\title{
High expression of GPNMB indicates an unfavorable prognosis in glioma: Combination of data from the GEO and CGGA databases and validation in tissue microarray
}

\author{
XIAO FENG ${ }^{1 *}$, LINA ZHANG ${ }^{2 *}$, SHANBAO KE ${ }^{3}$, TAO LIU $^{3}$, LIUWEI HAO ${ }^{4}$, \\ PAN ZHAO ${ }^{5}$, WENZHI TU ${ }^{6}$ and SHUNDONG CANG ${ }^{1}$
}

${ }^{1}$ Department of Oncology, ${ }^{2}$ Henan Provincial Key Laboratory of Kidney Disease and Immunology, Departments of ${ }^{3}$ Radiotherapy, ${ }^{4}$ Physical Examination and Health Management and ${ }^{5}$ Neurology, Henan Provincial People's Hospital, People's Hospital of Zhengzhou University, People's Hospital of Henan University, Zhengzhou, Henan 450003; ${ }^{6}$ Cancer Center, Shanghai General Hospital, Shanghai Jiao Tong University School of Medicine, Shanghai 201620, P.R. China

Received December 19, 2019; Accepted May 27, 2020

DOI: $10.3892 / \mathrm{ol} .2020 .11787$

\begin{abstract}
Glycoprotein non-metastatic melanoma protein B (GPNMB), a transmembrane glycoprotein, has been reported to be involved in tumor progression, but its prognostic value for glioma and the mechanistic effects on glioma progression have not been clearly explored. The present study aimed to investigate the prognostic role of GPNMB in glioma and the potential mechanisms of how GPNMB mediates glioma progression. Differentially expressed genes between the four highest and four lowest GPNMB expression samples in the GSE53733 dataset were first determined. Gene ontology, Kyoto Encyclopedia of Genes and Genomes pathway enrichment analysis and Gene set enrichment analysis results demonstrated that the significantly enriched pathways in samples with high GPNMB expression compared with those with low GPNMB expression were associated with hypoxia, angiogenesis, migration and invasion. Pearson correlation analysis was conducted to investigate the correlations between GPNMB expression and the markers of hypoxia, angiogenesis, migration and invasion in GSE53733, which were further
\end{abstract}

Correspondence to: Professor Shundong Cang, Department of Oncology, Henan Provincial People's Hospital, People's Hospital of Zhengzhou University, People's Hospital of Henan University, 7 Weiwu Road, Jinshui, Zhengzhou, Henan 450003, P.R. China E-mail: cangshundong@163.com

Dr Wenzhi Tu, Cancer Center, Shanghai General Hospital, Shanghai Jiao Tong University School of Medicine, 650 Xinsongjiang Road, Shanghai 201620, P.R. China

E-mail: wenzhitu@126.com

${ }^{*}$ Contributed equally

Key words: glycoprotein non-metastatic melanoma protein B, glioma, prognosis, bioinformatics analysis, tissue microarray validated using another mRNA microarray dataset from the Chinese Glioma Genome Atlas (CGGA). In addition, using the CGGA dataset, high GPNMB expression was demonstrated to be significantly associated with advanced WHO grade and short survival time in patients with glioma. Of note, based on the immunohistochemical staining of the tissue microarrays, Kaplan-Meier analysis with the Renyi test and a Cox proportional hazards model were used to validate the unfavorable prognostic role of high GPNMB expression in glioma. In conclusion, high GPNMB expression may be associated with high tumor grade and unfavorable prognosis in glioma. GPNMB expression was demonstrated to correlate with the markers of hypoxia, angiogenesis, migration and invasion, which may be potential mechanisms through which GPNMB mediates glioma progression.

\section{Introduction}

Glioma arises from glial or precursor cells of the central nervous system (CNS) and accounts for $\sim 26 \%$ of all primary CNS tumors and $>80 \%$ of malignant CNS tumors in the USA (1). Glioma has several histological subtypes, such as astrocytoma (including glioblastoma), oligodendroglioma and ependymoma among others $(1,2)$. Despite continuing progress in treatments (such as surgery, chemotherapy, radiotherapy, targeted therapy and immunotherapy), the prognosis of glioma, particularly that of glioblastoma, remains unfavorable. For example, the 1- and 5-year survival rates of glioblastoma in the USA from 2000-2014 were estimated to be 41.4 and $5.4 \%$, respectively (3). To date, although certain molecular alterations [e.g. isocitrate dehydrogenase 1 and 2 (IDH1/2) mutations] have been identified as prognostic markers for glioma $(4,5)$, more prognostic biomarkers are required due to the complex genomic alterations (6) and biological heterogeneity (7) of glioma.

Glycoprotein non-metastatic melanoma protein B (GPNMB), a type I transmembrane glycoprotein, was originally cloned from metastatic human melanoma cell lines (8). 
GPNMB is not only expressed in normal tissues (e.g. skin, bone, urinary system and CNS tissues), but also abnormally expressed in pathological tissues such as glaucoma, colitis, liver injury and a variety of carcinoma tissues (9), such as breast (10), gastric (11) or pancreatic cancer (12). GPNMB is located on the cell membrane and intracellular organelles, such as melanosomes and lysosomes, and can also be secreted into the extracellular compartment (13-15).

GPNMB serves multiple roles in both normal and tumor tissues. In normal tissues, GPNMB modulates various physiological processes, such as melanosome maturation (14), intercellular adhesion between keratinocytes and melanocytes (16), osteoclast and osteoblast differentiation $(15,17,18)$, and the regulation of immune responses $(19,20)$. Regarding the role of GPNMB in cancer, a variety of studies have demonstrated pro-tumorigenic roles of GPNMB in breast $(10,21)$, gastric (11), lung (22) and pancreatic cancer (12).

GPNMB has been demonstrated to be highly expressed in glioblastoma tissues (23) and to mediate glioma progression. Ono et al (24) have proposed that GPNMB prompts glioma progression by interacting with $\mathrm{Na}^{+} / \mathrm{K}^{+}$-ATPase $\alpha$ subunits Using in vitro assays, Bao et al (25) demonstrated that GPNMB mediated the proliferation and migration of glioma cells and tube formation of endothelial cells. These studies attributed the mechanisms of GPNMB-mediated glioma progression to one single molecule; however, the molecular mechanisms underlying the GPNMB-induced glioma progression may involve numerous pathways or complicated networks and remain insufficiently characterized. To date, the prognostic role of GPNMB in glioma has been inadequately studied, although an early study from Kuan et al (23) suggested that GPNMB was associated with increasing survival risk for patients with glioblastoma. However, due to the limited sample size, specific ethnicity and other confounding factors in their study, the prognostic role of GPNMB in glioma requires further investigation.

Thus, the present study aimed to comprehensively elucidate the potential mechanisms of GPNMB-induced glioma progression and identify multiple pathways through which GPNMB may mediate glioma progression via systemic bioinformatics analysis.

\section{Materials and methods}

Publicly available datasets. The GSE53733 dataset (26), which comprises the data of Affymetrix gene chip analyses from 70 German patients with glioblastoma, was downloaded from the GEO database (https://www.ncbi.nlm.nih. gov/geo/query/acc.cgi?acc=GSE53733). Another publicly available dataset, which was originally used in a study by Yan et al (27), contained mRNA microarray data and clinical information from 220 Chinese patients with glioma and was downloaded from the CGGA database (http://www.cgga.org. $\mathrm{cn} /$ ). The glioma patients in CGGA were classified into high and low GPNMB expression groups based on the median expression value of GPNMB.

Screening differentially expressed genes (DEGs). To preliminarily explore the disparity in transcriptome profiles between patients with high and low GPNMB expression, the four highest (GSM1299519, GSM1299555, GSM1299571, GSM1299574) and four lowest GPNMB expression samples (GSM1299575, GSM1299580, GSM1299583, GSM1299584) in GSE53733 were compared. The raw data from the GSE53733 dataset were processed using the R Project version 3.5.3 (https://www.r-project.org/) (28). The DEGs between the top and bottom 4 GPNMB expression samples were identified using the limma package in R Project (29). The screening thresholds for DEGs were set at adjusted P-value $=0.05$ and $\log _{2}$ fold-change $=2$.

Gene ontology $(G O)$ term and kyoto encyclopedia of genes and genomes (KEGG) pathway enrichment analysis. The upregulated DEGs in the four highest GPNMB expression samples compared with the four lowest samples were subjected to GO analysis using the Database for Annotation, Visualization and Integrated Discovery (https://david.ncifcrf. gov/). The enriched GO terms derived from the DEGs were categorized into three groups: Cell components (CC), molecular functions (MF) and biological processes (BP).

The enriched KEGG pathways of the DEGs were identified using the clusterProfiler package (30) and visualized using the pathview package (31) in R. The network diagram depicting complex interactions between significantly enriched KEGG pathways and DEGs was constructed using Cytoscape version 3.3.0 (https://cytoscape.org/).

Gene set enrichment analysis (GSEA). GSEA between the top and bottom four samples was conducted using GSEA (version 2.2.3; http://software.broadinstitute.org/gsea/downloads.jsp). Enrichment scores of 0-1 and nominal P-values for enriched gene sets were calculated using this software.

Correlation analysis. Correlation analysis was performed between GPNMB and markers of angiogenesis, migration and invasion, including cluster of differentiation 31 (CD31), endoglin (ENG), C-X-C motif chemokine receptor 4 (CXCR4), transforming growth factor $\beta 1$ (TGFB1), plasminogen activator, urokinase (PLAU), PLAU receptor (PLAUR) and matrix metalloproteinase 2 (MMP-2), MMP-7 and MMP-9. Pearson correlation analysis was used for parametric tests; Spearman correlation analysis was used for non-parametric tests. P-values for correlation analysis were determined using SPSS software (version 20.0.0; IBM Corp.).

Tissue microarray staining and scoring. The glioma tissue microarrays (G6042-3 and G6042-4) were purchased from Wuhan Servicebio Technology Co., Ltd. The tissue microarrays were subjected to immunohistochemical staining. Specifically, slides with tissue microarray were deparaffinized and rehydrated. Heat-induced antigen retrieval was conducted by immersing the slides into boiling Tris-EDTA buffer for $3 \mathrm{~min}$. Slides were incubated with $3 \%$ hydrogen peroxide for $30 \mathrm{~min}$ to block endogenous peroxidase and then blocked with blocking buffer (cat. no. P0102; Beyotime Institute of Biotechnology) for $1 \mathrm{~h}$ at room temperature. Slides were incubated with an anti-GPNMB primary antibody (1:500; cat. no. 38313; Cell Signaling Technology, Inc.) overnight at $4^{\circ} \mathrm{C}$. After washing the slides 3 times with Tris-Buffered Saline Tween-20 (TBST) for 5 min each time, 
the Real Envision Detection kit (cat. no. GK500710; GeneTech Biotechnology, Co., Ltd.) was used for signal visualization through the diaminobenzidine reaction. Nuclei were counterstained with hematoxylin. GPNMB immunoreactivity was scored by observing 3 random fields under a light microscope (Leica Microsystems, Inc.; magnification, x200). GPNMB immunoreactivity was scored based on staining distribution and intensity as previously described (23). According to the percentage of immunopositive cells, the staining distribution was categorized as follows: 0 points, $0 \%$; 1 point, $1-25 \%$; 2 points, $26-50 \%$; 3 points, $51-75 \%$; and 4 points, $76-100 \%$. The staining intensity was assessed and graded from 0 to 3 ( 0 points, negative; 1 point, weak staining; 2 points, moderate staining; and 3 points, strong staining). These two values were multiplied to achieve a maximum score of 12 . Scores of $0-6$ were considered low GPNMB expression, and scores of 7-12 were considered high GPNMB expression. The slides were evaluated by two independent observers. A total number of 74 Chinese patients with glioma were incorporated into the following analysis, after excluding those with incomplete follow-up information.

Cell culture and siRNA transfection. U-87MG (U87) glioblastoma cell line of unknown origin was obtained from the American Type Culture Collection (cat. no. HTB-14). U87 cells were cultured in Dulbecco's modified Eagle's medium (Thermo Fisher Scientific, Inc.) supplemented with $10 \%$ fetal bovine serum (FBS; Gibco; Thermo Fisher Scientific, Inc.). Small interfering RNA (siRNA) targeting human GPNMB (5'-GGATAATACTGGCCTGTTT-3') and a negative control siRNA (5'-GGCTCTAGAAAAGCC TATGC-3') were purchased from Guangzhou RiboBio, Co., Ltd. A total of $5 \times 10^{5}$ U87 cells were seeded in 6-well plates overnight and then transfected with $50 \mathrm{nM}$ siRNA using Lipofectamine ${ }^{\circledR} 2000$ (cat. no. 11668-019; Invitrogen; Thermo Fisher Scientific, Inc.) at $37^{\circ} \mathrm{C}$ for $6 \mathrm{~h}$ according to the manufacturer's instructions. Subsequent experiments were conducted $24 \mathrm{~h}$ post-transfection.

Western blotting. Cells were lysed for protein extraction with RIPA lysis buffer (cat. no. P0013B; Beyotime Institute of Biotechnology). Protein concentration was determined using an Enhanced BCA Protein Assay kit (cat. no. P0010; Beyotime Institute of Biotechnology). A total of $30 \mu \mathrm{g}$ protein for each sample was loaded per lane for sodium dodecyl sulfate-polyacrylamide gel electrophoresis using a $10 \%$ stacking gel to separate the proteins, which were subsequently transferred to $0.22-\mu \mathrm{m}$ PVDF membranes. The PVDF membranes were blocked with 5\% non-fat dry milk in Tris-buffered saline at room temperature for $1 \mathrm{~h}$, washed three times with Tris-Buffered Saline Tween-20 and incubated with primary antibodies against GPNMB (dilution, 1:1,000; cat. no. 38313; Cell Signaling Technology, Inc.) or GAPDH (dilution, 1:1,000; cat. no. 2118; Cell Signaling Technology, Inc.) overnight at $4^{\circ} \mathrm{C}$. The membranes were then washed three times and incubated with horseradish peroxidase-conjugated secondary antibodies (1:5,000; cat. no. 111-035-003; Jackson ImmunoResearch, Inc.) at room temperature for $1 \mathrm{~h}$. Finally, Immobilon $^{\mathrm{TM}}$ Western Chemiluminescent HRP Substrate (cat. no. P90719; EMD Millipore) was used to visualize signals.
Cell counting Kit-8 (CCK-8) assay. The CCK-8 assay (cat. no. CK04; Dojindo Molecular Technologies, Inc.) was performed in 96-well plates according to the manufacturer's instructions. A total of $3 \times 10^{3}$ U87 cell were plated in each well of a 96-well plate. Prior to measuring, $10 \mu \mathrm{l}$ of CCK-8 solution was added to each well and the plate was incubated for $2 \mathrm{~h}$ at $37^{\circ} \mathrm{C}$. The absorbance at $450 \mathrm{~nm}$ was measured on days 1-3, and 4 , respectively.

Cell migration and invasion assay. U87 migration and invasion assays were performed using 24-well Transwell plate inserts with $8-\mu \mathrm{m}$ pores (cat. no. 3422; Corning, Inc.). For the cell invasion assay, the inserts were pre-coated with Matrigel (cat. no. 356234; Corning, Inc.) at $4^{\circ} \mathrm{C}$ and incubated at $37^{\circ} \mathrm{C}$ for $2 \mathrm{~h}$. A total of $600 \mu \mathrm{l}$ DMEM supplemented with $10 \%$ FBS was added into the lower chamber, and $200 \mu 1$ serum-free medium containing $5 \times 10^{5}$ U87 cells was added into the upper chamber. Following 24-h incubation, U87 cells in the upper chamber were removed with cotton swabs. Migratory or invasive U87 cells were fixed with $4 \%$ paraformaldehyde at room temperature for $1 \mathrm{~h}$ and stained with $0.1 \%$ crystal violet at room temperature for $15 \mathrm{~min}$. The number of migratory or invasive U87 cells was determined by counting cells from five random fields under a light microscope (Leica Microsystems, Inc.) with x200 magnification.

Statistical analysis. Data are presented as the mean \pm standard error of the mean. In CCK- 8 assay, 6 independent repeats for each group were performed and in the cell migration and invasion assays, 5 random fields were observed for cell counting. Statistical differences were calculated using SPSS Statistics (version 20; IBM, Inc.) and GraphPad Prism (version 6.02; GraphPad Software, Inc.). For parametric tests, unpaired Student's t-test was used for two-group comparisons, and one-way ANOVA followed by Tukey's post hoc test was conducted for multi-group comparisons. For non-parametric tests, Mann-Whitney U test was performed for two-group comparisons, and Kruskal-Wallis test followed by Dunn's multiple comparisons test was used. The Kaplan-Meier method followed by log-rank test was used for survival analysis. The Renyi test was performed to detect differences when survival curves crossed over with the survMisc package in R. The Cox proportional hazards model was used for univariate and multivariate analysis. $\mathrm{P}<0.05$ was considered to indicate a statistically significant difference.

\section{Results}

Screening DEGs between samples with high and low GPNMB expression, and hierarchical clustering analysis. The four highest and four lowest GPNMB expression samples in the GSE53733 dataset were compared to screen for DEGs. Based on the aforementioned screening thresholds, 254 up- and 79 downregulated DEGs were identified and are presented in the volcano plot (Fig. 1A). Hierarchical clustering analysis was performed using the top 50 up- and downregulated DEGs between the four highest and four lowest GPNMB expression samples in GSE53733. The top 50 up- (Fig. 1B) and downregulated (Fig. 1C) DEGs are presented in the heat maps. 


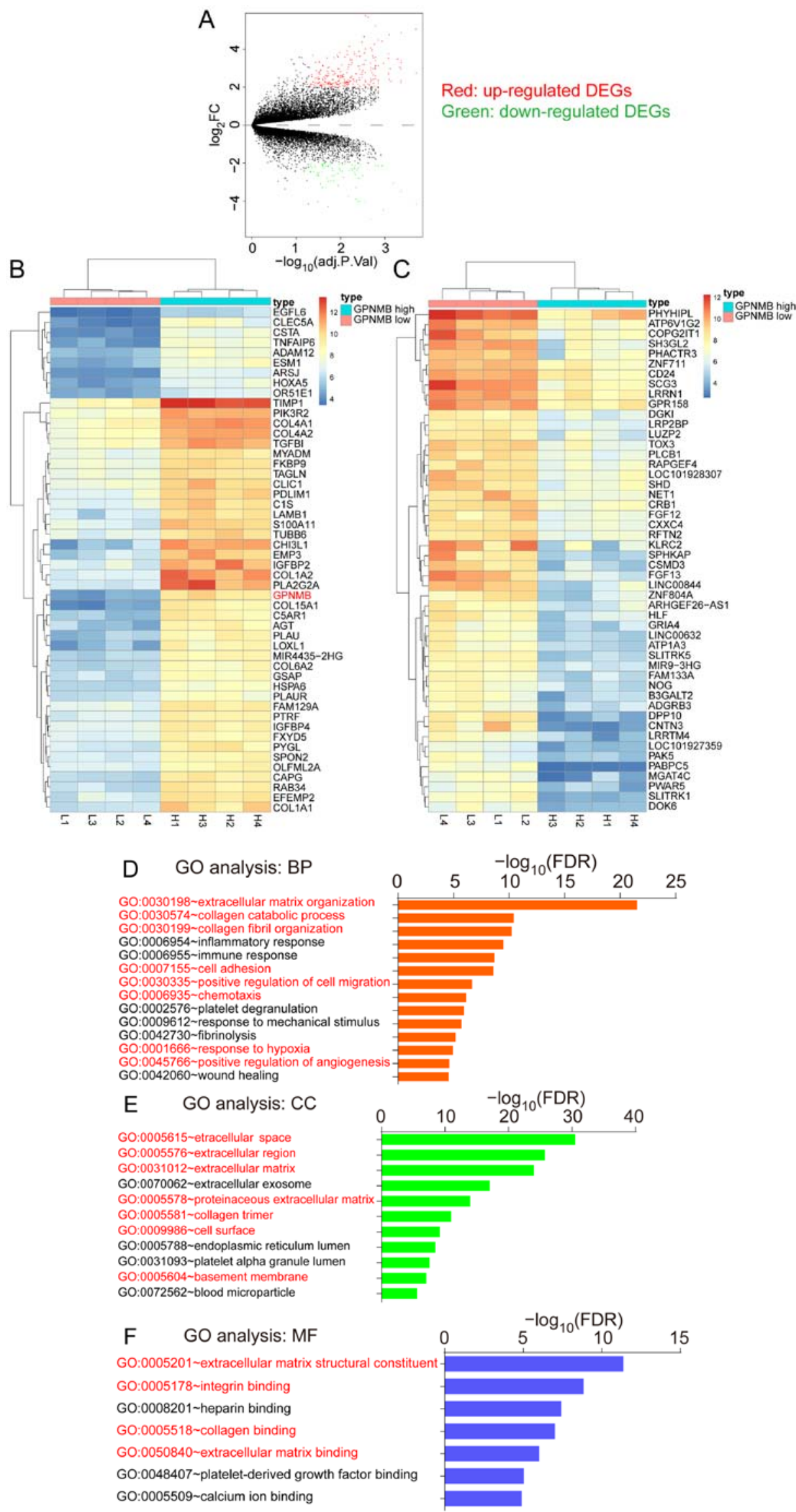

Figure 1. Hierarchical clustering and GO analysis of the DEGs between the four highest and four lowest GPNMB expression samples. (A) Volcano plot of the DEGs (screening thresholds: Adj.P.Val=0.05; $\log _{2}$ fold-change=2). Red spots represent the upregulated DEGs and green spots represent the downregulated DEGs in the high GPNMB expression group. (B and C) Heat maps of (B) the top 50 up- and (C) top 50 downregulated DEGs. (D-F) GO analysis revealed significantly enriched pathways of the upregulated DEGs in (D) BP, (E) CC and (F) MF. DEGs, differentially expressed genes; Adj.P.Val, adjusted P-value; GPNMB, glycoprotein non-metastatic melanoma protein B; GO, gene ontology; BP, biological process; CC, cellular component; MF, molecular function. 
A

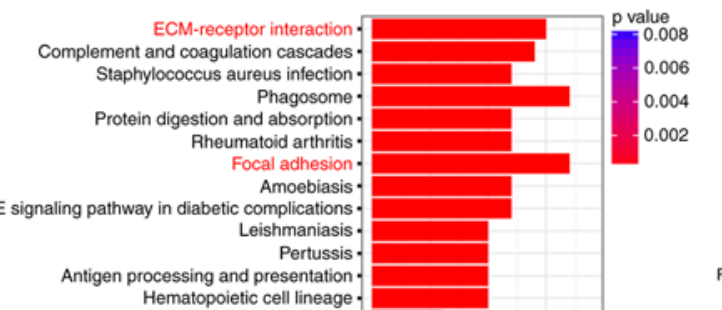

B
Up-regulated DEGs
Down-regulated DEGs
Significantly enriched KEGG pathways

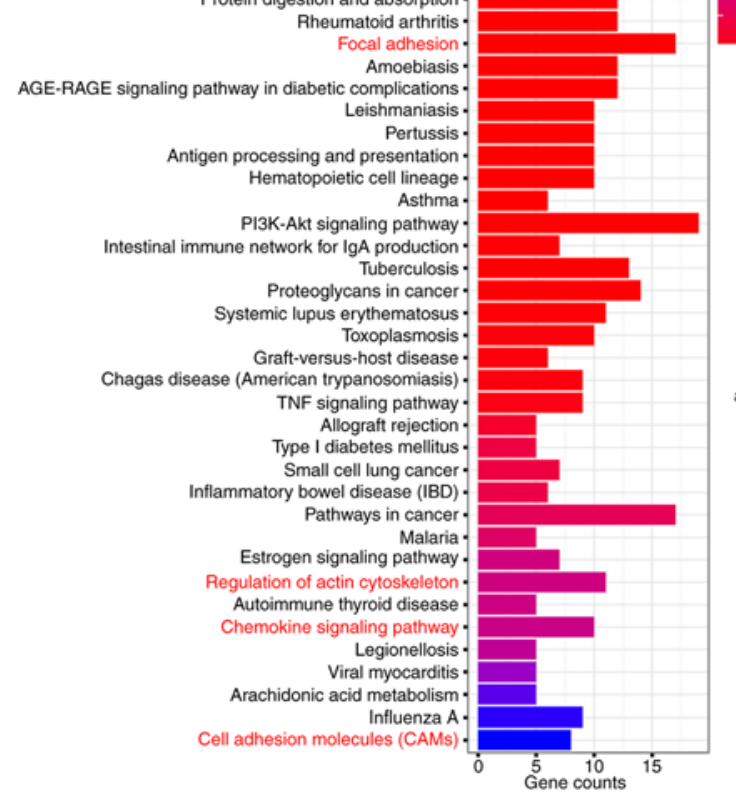

\subsection{2}

AGE-RAGE

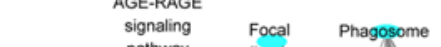

Regulation diabettc Malara toxoplasmosis $\begin{aligned} & \text { Chagas Legionellosis } \\ & \text { disease } \\ & \text { (American Pertussis Estrogen Complement }\end{aligned}$

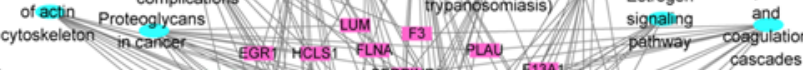

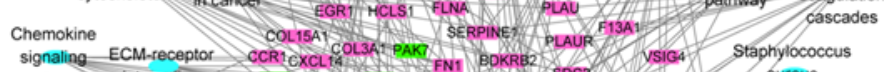

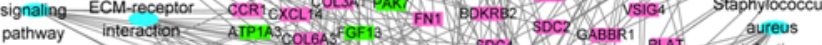

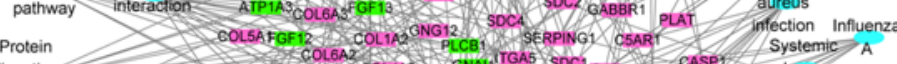

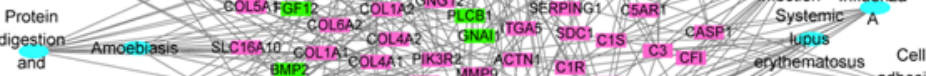

absorption Leishmaniasis COL5A2 TBSP

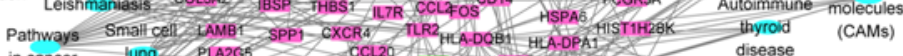

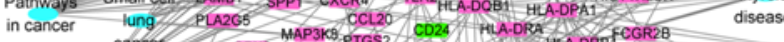

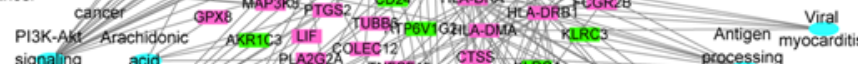

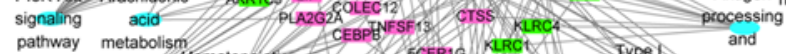
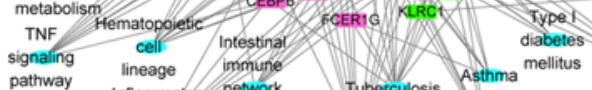

lignaling lineage immune
Inflammatory network Tuberculosis Asthma mellitus Allograft

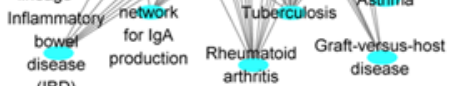

(IBD)

\section{C}

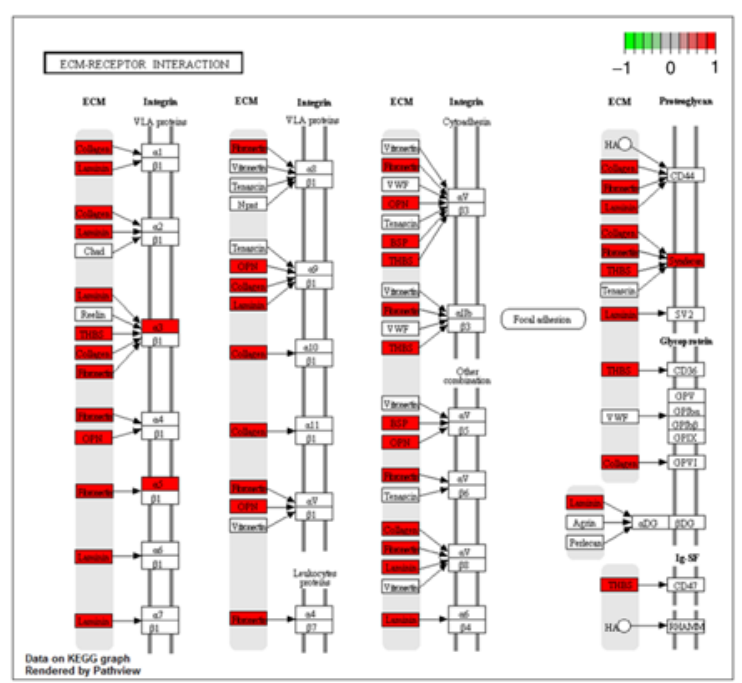

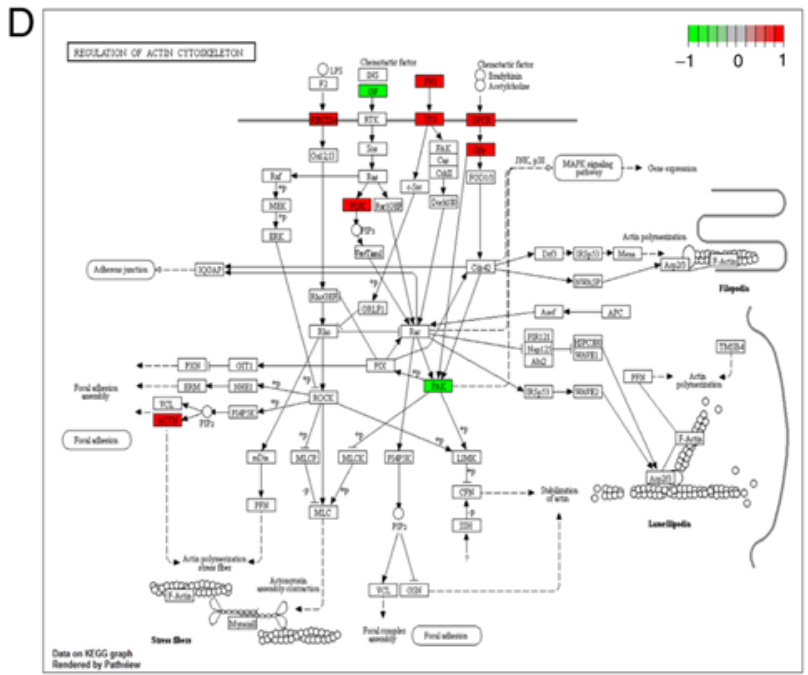

Figure 2. KEGG pathway enrichment analysis of DEGs. (A) Significantly enriched KEGG pathways of the DEGs. (B) Network diagram of the complex interactions among the DEGs and the significantly enriched KEGG pathways. (C) KEGG pathway of 'ECM-receptor interaction'. (D) KEGG pathway of 'actin cytoskeleton'. KEGG, Kyoto Encyclopedia of Genes and Genomes; DEGs, screening differentially expressed genes; ECM, extracellular matrix.

$G O$ and KEGG analysis of DEGs. GO pathways in which the 79 downregulated DEGs were enriched were irrelevant to tumor biological behaviors and were therefore not studied (data not shown). The 254 upregulated DEGs were subjected to GO analysis. The significantly enriched GO terms in BP comprised 'extracellular matrix organization', 'collagen catabolic process', 'collagen fibril organization', 'inflammatory response' etc. (Fig. 1D). The significantly enriched GO terms in CC included 'extracellular space', 'extracellular region', extracellular matrix', 'extracellular exosome'etc. (Fig. 1E). The significantly enriched GO terms in MF contained 'extracellular matrix structural constituent', 'integrin binding', 'heparin binding', 'collagen binding'etc. (Fig. 1F).

KEGG analysis using DEGs identified 38 significantly enriched pathways (Fig. 2A). To illustrate the interactions between the DEGs and the enriched KEGG pathways, a network diagram was constructed (Fig. 2B). The 'ECM-receptor interaction' and the 'regulation of actin cytoskeleton' are vital pathways mediating cell migration and invasion and are presented in detail in Fig. 2C and D.

GSEA analysis. GSEA analysis between the four highest and four lowest GPNMB expression samples in GSE53733 identified the following significantly enriched pathways: 'Hypoxia' (Fig. 3A), 'angiogenesis' (Fig. 3B), 'epithelial mesenchymal transition' (Fig. 3C), 'focal adhesion' (Fig. 3D), 'chemokine receptors bind chemokines' (Fig. 3E), 'chemokine receptor binding' (Fig. 3F), 'ECM component' (Fig. 3G), 'ECM binding' (Fig. 3H) and 'ECM receptor interaction' (Fig. 3I).

Correlation analysis between GPNMB and markers of angiogenesis, migration and invasion using the GSE53733 dataset 

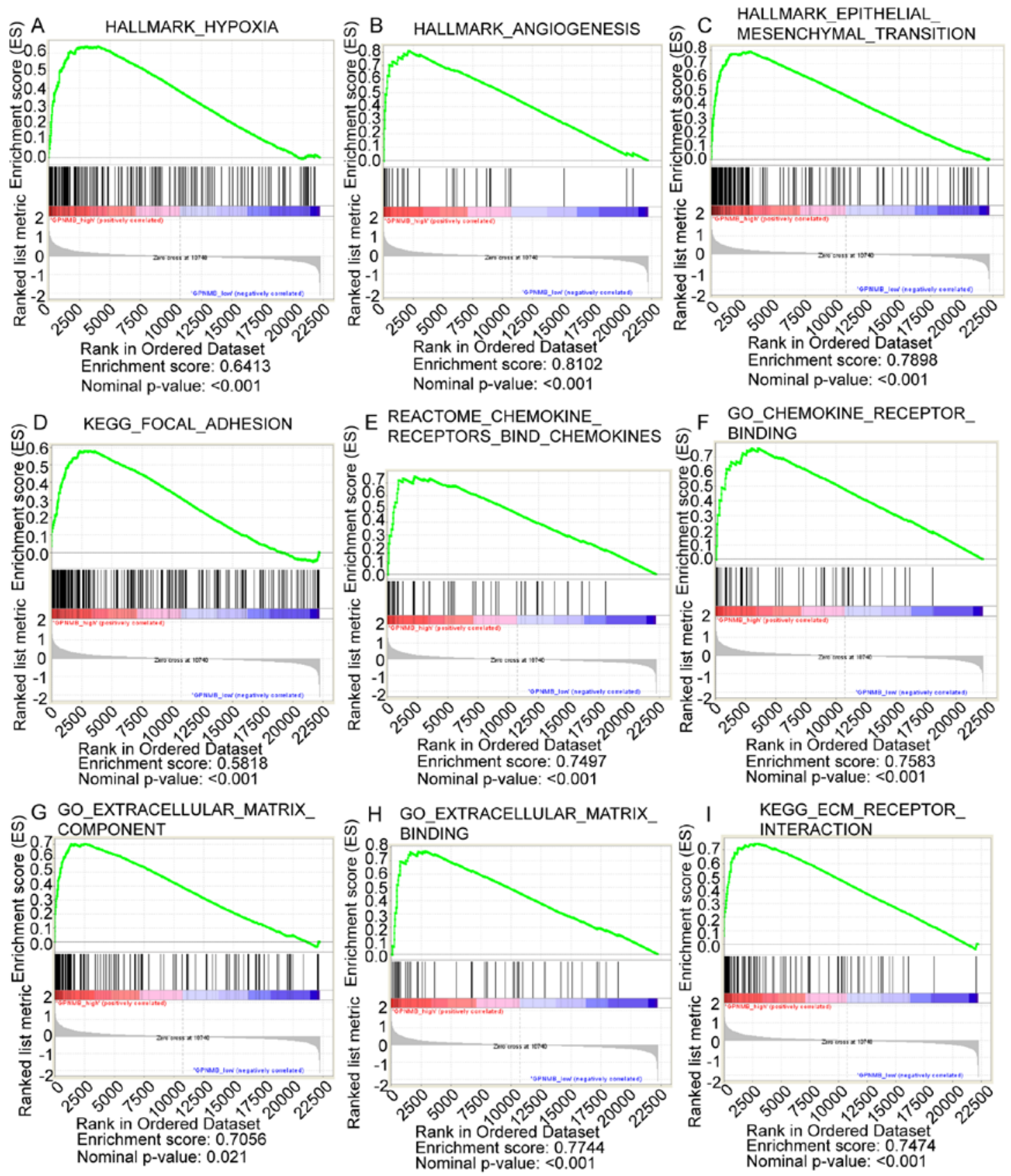

Figure 3. Gene Set Enrichment Analysis between the four highest and four lowest GPNMB expression samples in GSE53733. (A-I) Enriched pathways of (A) 'hypoxia', (B) 'angiogenesis', (C) 'epithelial mesenchymal transition', (D) 'focal adhesion', (E) 'chemokine receptors bind chemokines', (F) 'chemokine receptor binding', (G) 'extracellular matrix component', (H) 'extracellular matrix binding' and (I) 'extracellular matrix receptor interaction'. GPNMB, glycoprotein non-metastatic melanoma protein B.

and validation in the CGGA dataset. The GO and $\mathrm{KEGG}$ pathway analysis, as well as GSEA results revealed that DEGs between high and low GPNMB expression samples were generally enriched in pathways associated with angiogenesis, migration and invasion. Therefore, the present study subsequently investigated whether GPNMB expression correlated with the expression of the known markers of angiogenesis, migration and invasion. In the GSE53733 dataset, Pearson correlation analysis determined that GPNMB expression was positively correlated with the expression of CD31 $(r=0.548$; $\mathrm{P}<0.001)$, ENG $(\mathrm{r}=0.465 ; \mathrm{P}<0.001$; weak positive correlation), CXCR4 ( $r=0.584 ; \mathrm{P}<0.001)$, TGFB1 ( $\mathrm{r}=0.390 ; \mathrm{P}<0.001$; weak positive correlation), PLAU ( $r=0.557$; $\mathrm{P}<0.001)$, PLAUR
( $\mathrm{r}=0.657 ; \mathrm{P}<0.001)$, MMP-2 ( $\mathrm{r}=0.396 ; \mathrm{P}<0.001$; weak positive correlation), MMP-7 ( $\mathrm{r}=0.341 ; \mathrm{P}<0.001$; weak positive correlation) and MMP-9 ( $\mathrm{r}=0.496 ; \mathrm{P}<0.001$; weak positive correlation) (Fig. 4). Using a validation dataset from CGGA, Spearman correlation analysis revealed significant correlations between the expression of GPNMB and CD31 ( $\mathrm{rho}=0.684 ; \mathrm{P}<0.001$ ), ENG (rho=0.502; $\mathrm{P}<0.001)$, CXCR4 (rho $=0.661 ; \mathrm{P}<0.001)$, TGFB1 (rho=0.334; $\mathrm{P}<0.001$ ), PLAU ( $r h o=0.631 ; \mathrm{P}<0.001$ ), PLAUR (rho=0.619; $\mathrm{P}<0.001$ ), MMP-2 (rho=0.409; $\mathrm{P}<0.001$ ), MMP-7 (rho $=0.455 ; \mathrm{P}<0.001)$ and MMP-9 $(\mathrm{rho}=0.518$; $\mathrm{P}<0.001$ ) (Fig. 5).

In addition, correlation analysis revealed that GPNMB expression was positively associated with CD163 in the 

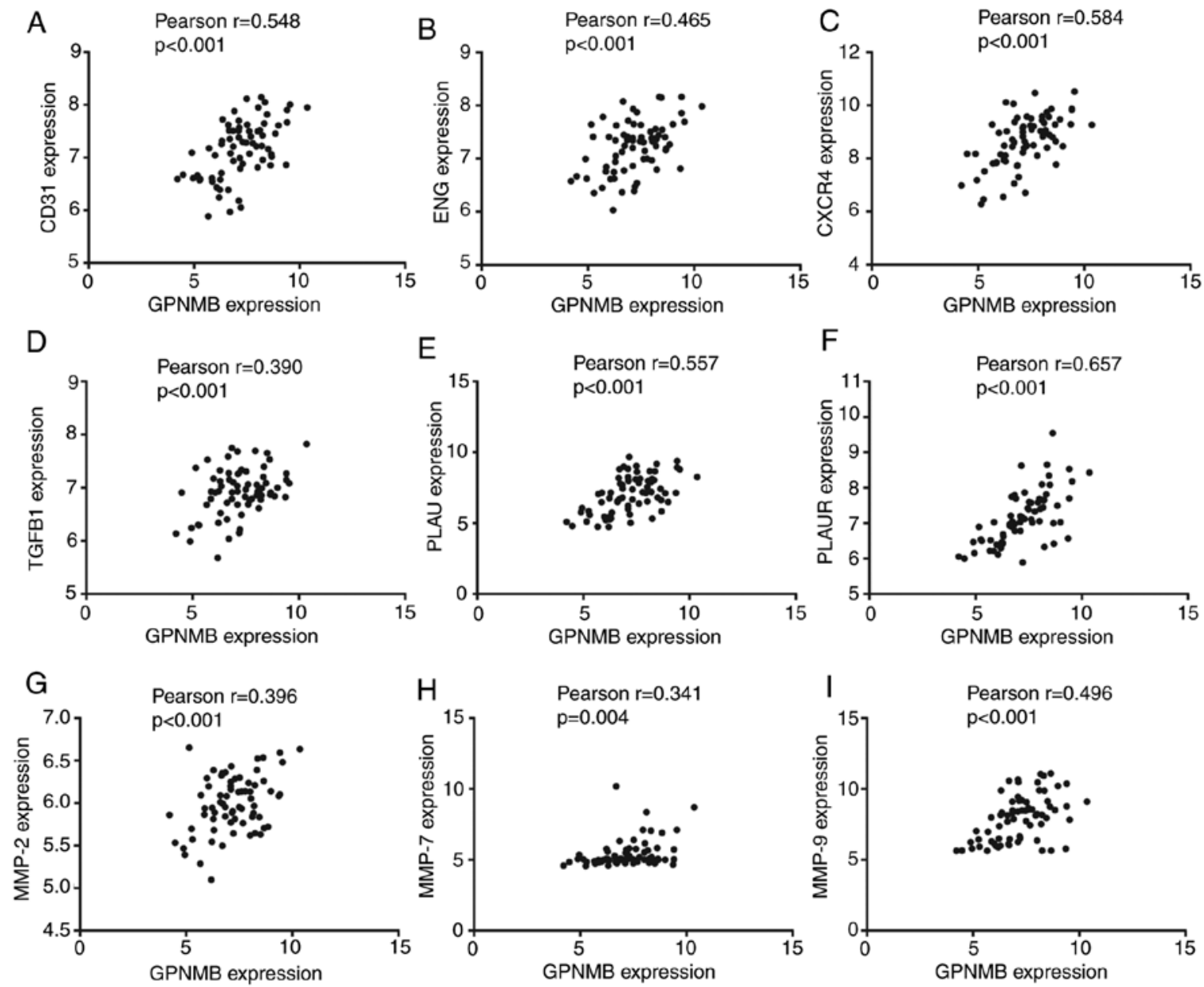

Figure 4. Pearson correlation analysis between GPNMB and markers of angiogenesis, migration and invasion in GSE53733. (A-I) Correlations between GPNMB and (A) CD31, (B) ENG, (C) CXCR4, (D) TGFB1, (E) PLAU, (F) PLAUR, (G) MMP-2, (H) MMP-7 and (I) MMP-9. GPNMB, glycoprotein non-metastatic melanoma protein B; CD31, cluster of differentiation 31; ENG, endoglin; CXCR4, C-X-C motif chemokine receptor 4; TGFB1, transforming growth factor $\beta 1$; PLAU, urokinase; PLAUR, PLAU receptor; MMP, matrix metalloproteinase.

GSE53733 dataset $(\mathrm{r}=0.671 ; \mathrm{P}<0.001 ;$ Fig. S1A) and the microarray dataset in CGGA (rho=0.670; $\mathrm{P}<0.001$; Fig. S1B).

In vitro experiments investigating the effects of GPNMB on glioma cell proliferation, migration and invasion. Fig. S2A demonstrates the knockdown of GPNBM in U87 cells transfected with siRNA. Subsequent experiments revealed that knockdown of GPNBM significantly inhibited the proliferation, migration and invasion of U87 cells (Fig. S2B-D).

Clinical and prognostic role of GPNMB expression in glioma. Using the mRNA microarray data and clinical information of the 220 Chinese patients with glioma from the CGGA, the clinical and prognostic role of GPNMB expression in glioma was investigated. Compared with non-tumor brain tissues, higher levels of GPNMB expression were observed in glioma tissues (Fig. 6A). The four gene expression-based molecular subtypes (proneural, neural, classical and mesenchymal) of glioblastoma were previously identified and characterized (32). The highest levels of GPNMB expression were identified in patients with the mesenchymal subtype (Fig. 6B). Regarding the association between histological subtypes and GPNMB expression, the highest levels of GPNMB expression were detected in patients with glioblastoma (Fig. 6C). GPNMB expression was higher in high-grade glioma (WHO III or IV) compared with that in low-grade glioma (WHO II) (Fig. 6D). Lower Karnofsky performance scores were observed in patients with high GPNMB expression compared with those with low expression (Fig. 6E). Kaplan-Meier analysis with the log-rank test demonstrated that high GPNMB expression was associated with a shorter survival time $(\mathrm{P}<0.001$; Fig. $6 \mathrm{~F})$. Survival analysis in sub-populations stratified by age revealed that high GPNMB expression was significantly associated with a lower survival rate in patients aged $31-50$ years $(\mathrm{P}<0.001$; Fig. $6 \mathrm{H})$ and 51-70 years $(\mathrm{P}=0.017$; Fig. 6I), but not in patients aged $10-30$ years $(\mathrm{P}=0.954$; Fig. $6 \mathrm{G})$.

To validate the prognostic role of GPNMB in glioma, tissue microarrays were immunohistochemically stained with an anti-GPNMB antibody. Microarrays from 74 Chinese patients with glioma with complete follow-up information were analyzed. Representative images of GPNMB staining with different intensity scores are presented in Fig. 7A. No significant differences in GPNMB expression distribution were observed between female and male patients $\left(\chi^{2}=0.173 ; \mathrm{P}=0.678\right.$; Table I). The proportion of patients with high GPNMB expression was higher in patients aged $>40$ years compared with that in patients aged $\leq 40$ years $\left(\chi^{2}=5.316 ; \mathrm{P}=0.021\right.$; Table I). The GPNMB expression level was significantly correlated 
Table I. Association of GPNMB expression with clinical parameters in the 74 glioma patients.

\section{GPNMB expression}

Variable

Low

High

Statistics value

P-value

Sex

$\begin{array}{lrrr}\text { Female } & 17 & 7 & 0.173\left(\chi^{2}\right) \\ \text { Male } & 33 & 17 & \\ \text { Age } & & & 5.316\left(\chi^{2}\right) \\ \leq 40 & 22 & 4 & 0.678 \\ >40 & 28 & 20 & 0.353 \text { (Spearman rho })^{\mathrm{a}} \\ \text { WHO grade } & & & \\ \text { I } & 1 & 0 & 0.002^{\mathrm{a}} \\ \text { II } & 20 & 4 & \\ \text { III } & 18 & 6 & \\ \text { IV } & 11 & 14 & \end{array}$

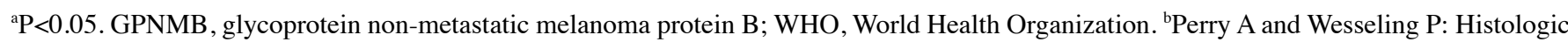
classification of gliomas. Handbook of clinical neurology 134: 71-95, 2016.

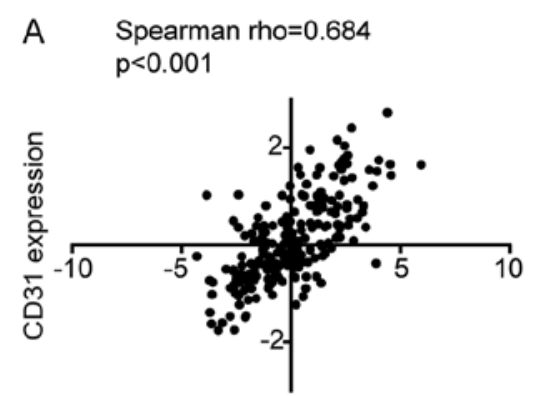

GPNMB expression
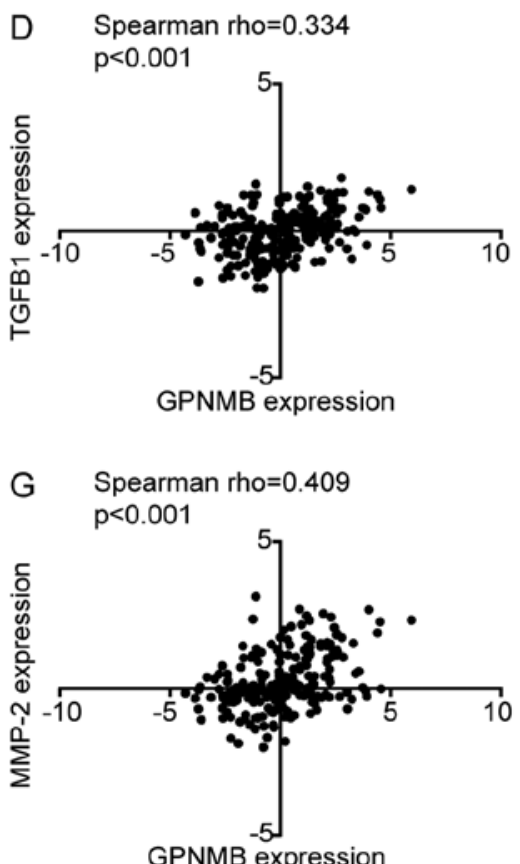
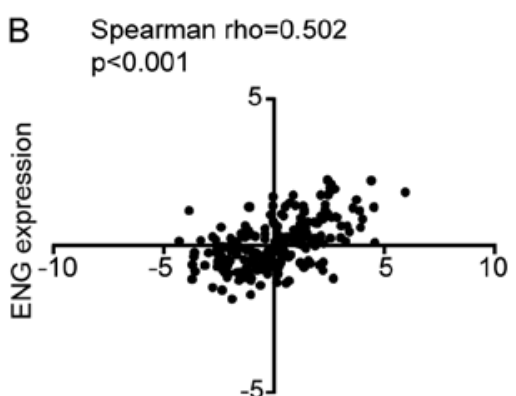

GPNMB expression
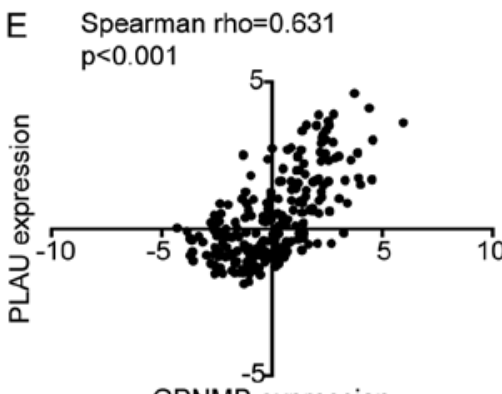

GPNMB expression

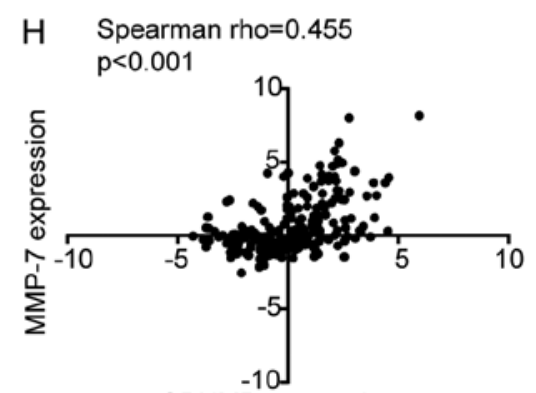

GPNMB expression

\section{Spearman $r$ ho $=0.661$}

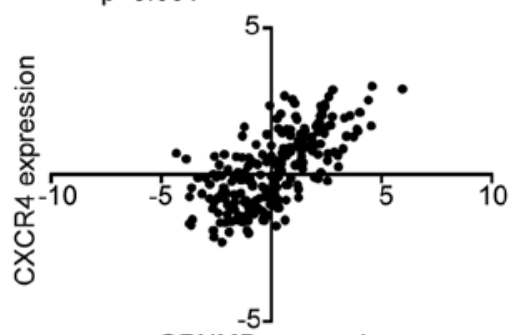

GPNMB expression
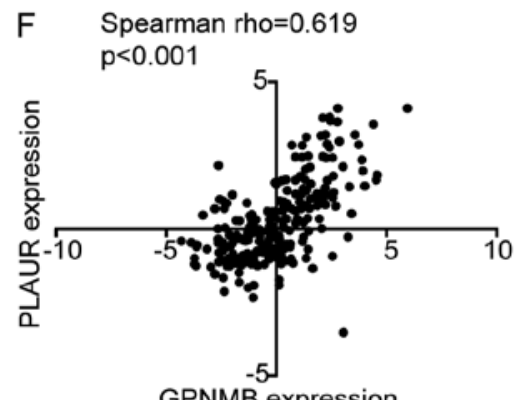

GPNMB expression

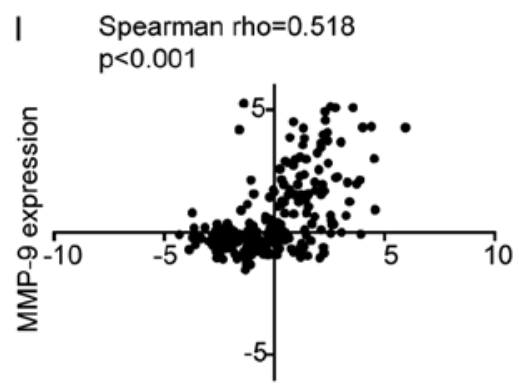

GPNMB expression

Figure 5. Spearman correlation analysis between GPNMB and markers of angiogenesis, migration and invasion in the mRNA microarray dataset in Chinese Glioma Genome Atlas. (A-I) Correlations between GPNMB and (A) CD31, (B) ENG, (C) CXCR4, (D) TGFB1, (E) PLAU, (F) PLAUR, (G) MMP-2, (H) MMP-7 and (I) MMP-9. GPNMB, glycoprotein non-metastatic melanoma protein B; CD31, cluster of differentiation 31; ENG, endoglin; CXCR4, C-X-C motif chemokine receptor 4; TGFB1, transforming growth factor $\beta 1$; PLAU, urokinase; PLAUR, PLAU receptor; MMP, matrix metalloproteinase. 
A

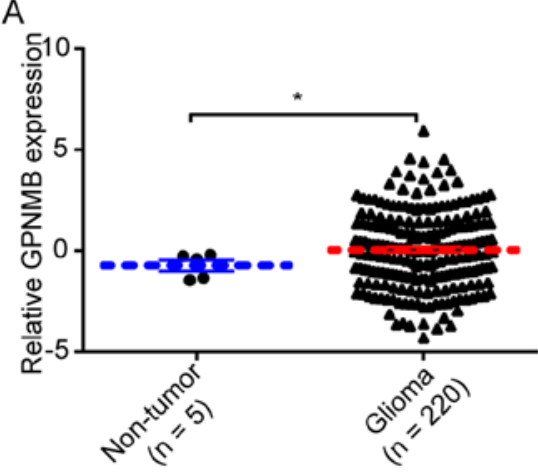

D

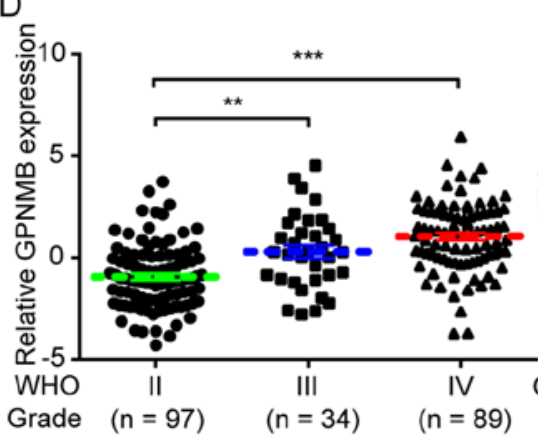

G

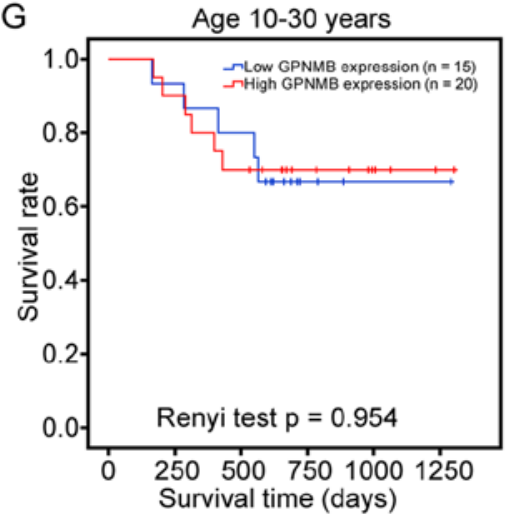

$\mathrm{B}$

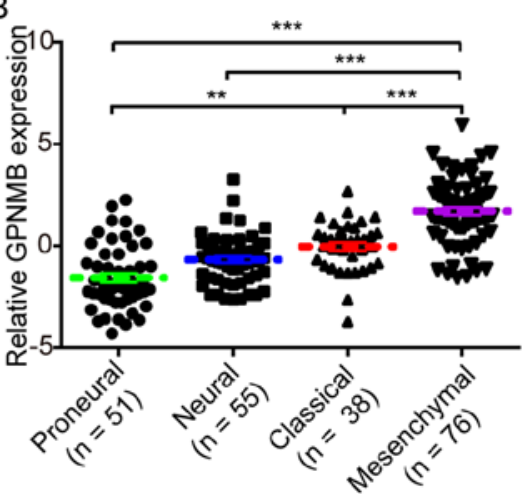

E

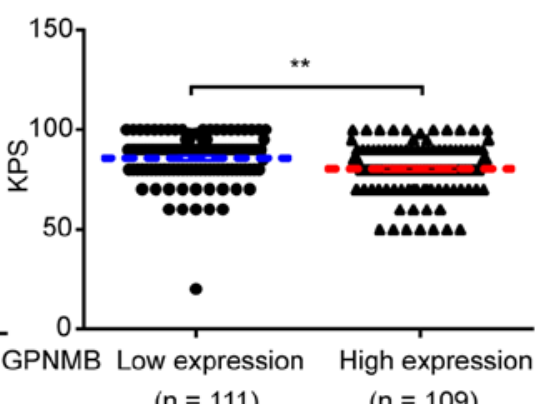

$(\mathrm{n}=111)$

$(n=109)$

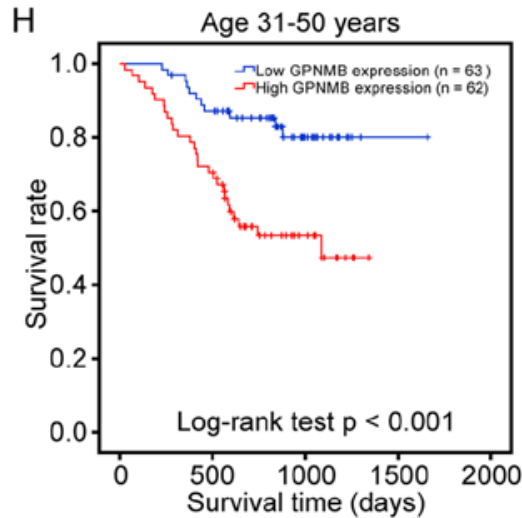

\section{C}

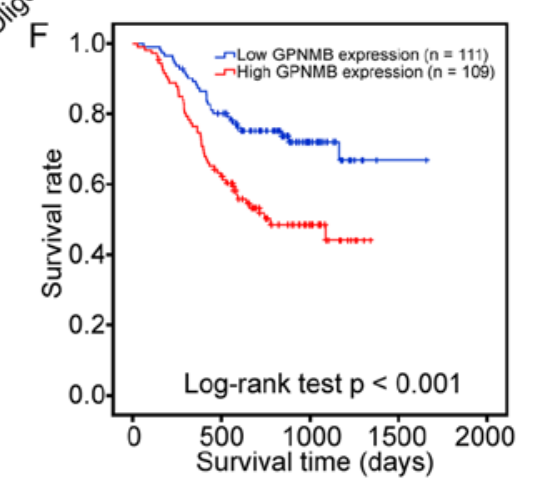

I

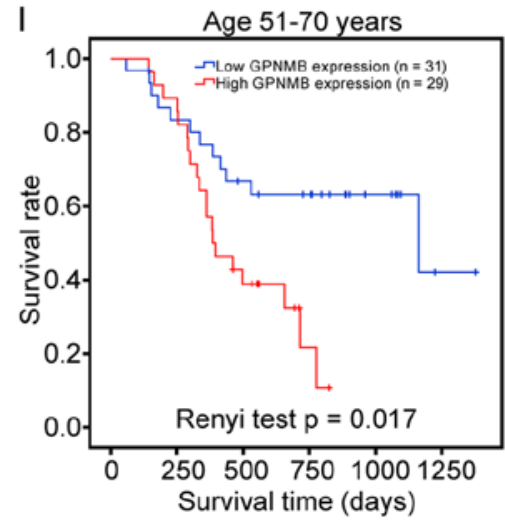

Figure 6. The association between GPNMB expression and clinicopathological characteristics of 220 patients with glioma from the Chinese Glioma Genome Atlas dataset explored using the mRNA microarray data and clinical information. (A) Compared with non-tumor brain tissues, higher levels of GPNMB expression were observed in glioma tissues. (B and C) The GPNMB expression levels were compared in the (B) four gene expression-based and (C) four histological subtypes. (D) The levels of GPNMB expression in different WHO grades were compared. (E) Lower KPS were identified in patients with high GPNMB expression. (F) Kaplan-Meier analysis with the log-rank test demonstrated that high GPNMB expression was associated with shorter survival time compared with that of patients with low GPNMB expression. (G and H) Survival differences were compared between patients with high and low GPNMB expression in sub-populations aged (G) 10-30, (H) 31-50 and (I) $51-70$ years. ${ }^{*} \mathrm{P}<0.05,{ }^{* *} \mathrm{P}<0.01$ and ${ }^{* * *} \mathrm{P}<0.001$. GPNMB, glycoprotein non-metastatic melanoma protein B; KPS, Karnofsky performance scores.

with the WHO grade (rho=0.353; $\mathrm{P}=0.002$; Table I), and the GPNMB expression scores were higher in high-compared with low-grade glioma ( $\mathrm{P}=0.018$; Fig. 7B). Survival analysis using Renyi test demonstrated that high GPNMB expression was significantly associated with a lower survival rate $(\mathrm{P}=0.002$; Fig. 7C). In addition, multivariate analysis based on the Cox proportional hazards model revealed that GPNMB expression was an independent prognostic factor for glioma $(\mathrm{P}=0.009$, Table II).

\section{Discussion}

To date, although a number of predictive molecular markers (e.g., 1p/19q co-deletion and IDH1/2 mutations) have been identified as prognostic markers for glioma (33-35), more are urgently required, partly due to the great complexity of the molecular traits of glioma (36). GPNMB, a transmembrane glycoprotein, has been demonstrated to be involved in tumor progression $(11,12,21,22)$, whereas its mechanistic effects on glioma progression and its prognostic role have not been thoroughly investigated. Therefore, the aim of the present study was to elucidate the prognostic role of GPNMB in glioma through data mining of publicly accessible datasets through validation by immunohistochemical staining of a tissue microarray. Systematic bioinformatics analysis was conducted to explore the molecular disparities between samples with high and low GPNMB expression; the results identified potential mechanisms through which GPNMB may mediate glioma progression. 
Table II. Univariate and multivariate analysis based on Cox proportional hazards model explored prognostic role of GPNMB.

\begin{tabular}{lccrr}
\hline & & \multicolumn{2}{c}{ Multivariate analysis } \\
\cline { 2 - 5 } Variable & Univariate analysis P-value & P-value & RR & $95 \%$ CI \\
\hline Sex (female vs. male) & 0.281 & 0.181 & 0.637 & $0.330-1.232$ \\
Age ( $\leq 40$ vs. $>40)$ & 0.702 & 0.409 & 1.340 & $0.669-2.686$ \\
WHO grade (I and II vs. III and IV) & $0.043^{\mathrm{a}}$ & 0.114 & 0.562 & $0.275-1.148$ \\
GPNMB expression (low vs. high) & $0.005^{\mathrm{a}}$ & $0.009^{\mathrm{a}}$ & 0.413 & $0.213-0.803$ \\
\hline
\end{tabular}

${ }^{\text {a }}<<0.05$. RR, risk ratio; CI, confidence interval; GPNMB, glycoprotein non-metastatic melanoma protein B; WHO, World Health Organization. ${ }^{b}$ Perry A and Wesseling P: Histologic classification of gliomas. Handbook of clinical neurology 134: 71-95, 2016.

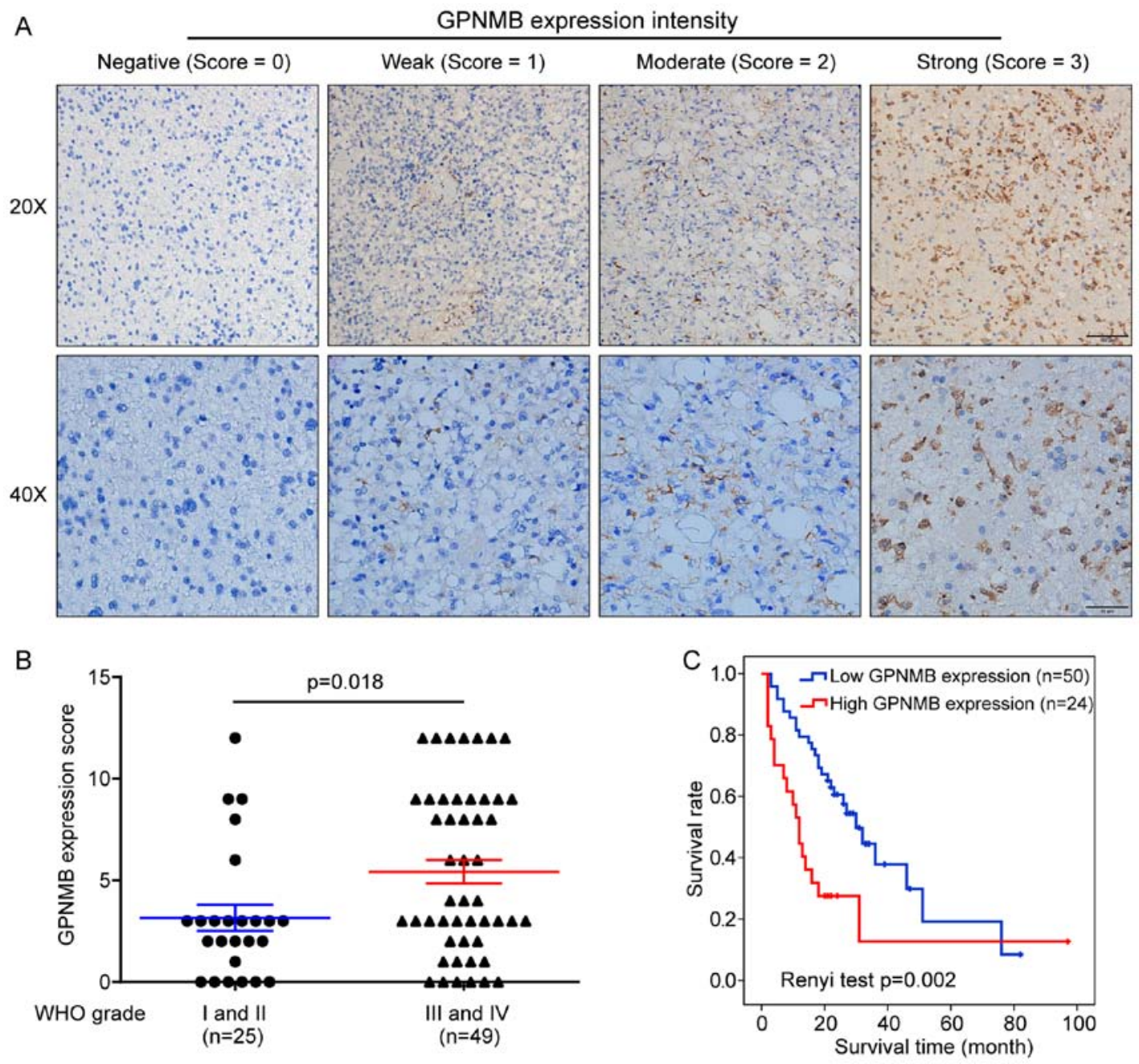

Figure 7. Prognostic role of GPNMB in glioma confirmed in tissue microarrays. (A) Representative images of immunohistochemical staining against GPNMB with different intensity scores. (B) The immunohistochemical scores of GPNMB expression were higher in high-grade (WHO III and IV) compared with low-grade (WHO I and II) glioma. (C) The Renyi test demonstrated that high GPNMB expression was significantly associated with a lower survival rate compared with that in the low GPNMB expression group $(\mathrm{P}=0.002)$. GPNMB, glycoprotein non-metastatic melanoma protein $\mathrm{B}$; WHO, World Health Organization.

In the present study, the upregulated DEGs in samples with high GPNMB expression compared with samples with low GPNMB expression were subjected to GO enrichment analysis. The processes of cell migration and invasion have been established to be inextricably linked with and characterized by the extracellular matrix (ECM), cell adhesion, cell cytoskeleton and chemotaxis (37-41). In the present study, the most significantly enriched GO pathways participated in migration and invasion. In addition, KEGG analysis results consistently revealed that the DEGs were significantly enriched in pathways associated with cell migration and invasion, such as 'ECM-receptor interaction', 'focal adhesion', 'regulation of 
actin cytoskeleton', 'chemokine signaling pathway' and 'cell adhesion molecules'. The subsequent GSEA demonstrated that glioma samples with high GPNMB expression were characterized by enriched pathways involved in migration and invasion. Correlation analysis was performed to explore the relationship between GPNMB and markers of migration and invasion. GPNMB expression was positively correlated with that of CXCR4, TGFB1, PLAU, PLAUR, MMP-2, MMP-7 and MMP-9, which are considered to be markers of tumor cell migration and invasion (42-51). Taken together, the comprehensive bioinformatics analysis, which included GO, KEGG, GSEA and correlation analysis, indicated the potential pathways through which GPNMB promotes glioma migration and invasion.

To the best of our knowledge, studies concerning the effects of GPNMB on glioma angiogenesis are rare. Using in vitro assays, Bao et al (25) demonstrated that GPNMB knockdown in glioma cells suppressed the tube formation of endothelial cells induced by conditioned medium from glioma cells. Considering that glioma angiogenesis is a highly complicated in vivo process (52), evidence from Bao et al (25) for the role of GPNMB in glioma angiogenesis may be reasonable but inadequate. In the present study, GO analysis revealed that the upregulated DEGs in samples with high GPNMB expression were enriched in 'response to hypoxia' and 'positive regulation of angiogenesis'. Consistently, GSEA revealed that samples with high GPNMB expression were characterized by hypoxia and angiogenesis. In addition, GPNMB was positively correlated with CD31 and ENG, which are markers of angiogenesis $(53,54)$. Taken together, based on systemic bioinformatics analysis, the results of the present study suggested that the effects of GPNMB on angiogenesis may be crucial mechanisms of glioma progression.

In addition to the effects of GPNMB on glioma angiogenesis, migration and invasion, several recent studies have suggested other potential mechanisms of GPNMB-mediated glioma progression which involve glioma-associated microglia/macrophage (GAM) polarization towards the M2 phenotype (55-57). GAM with the M2 phenotype has protumoral and immunosuppressive properties $(58,59)$. Thus, the present study explored the correlation between GPNMB and CD163 (a known marker for GAM with the M2 phenotype) expression (60). In the present study, the correlation analysis revealed that GPNMB expression was positively associated with CD163 in the GSE53733 dataset and the microarray dataset in CGGA. In addition, GPNMB expression was significantly correlated with that of TGFB1, which is another known marker for GAM polarization towards the M2 phenotype (60). Although the correlation analysis indicated positive associations between GPNMB and markers of M2-phenotype GAM, the specific roles of GPNMB in GAM polarization require more thorough investigation and solid evidence.

To further validate the effects of GPNMB on glioma progression, in vitro experiments were performed to determine the role of GPNMB in glioma cell proliferation, migration and invasion. Fig. S2 demonstrates that knockdown of GPNBM significantly inhibited the proliferation, migration and invasion of U87 cells. These in vitro experiments consolidated the tumor-promoting role of GPNBM in glioma progression.
The prognostic role of GPNMB in glioma is not fully determined, although an early study from Kuan et al (23) suggested that GPNMB was associated with higher risk of death. Due to the limited sample size in survival analysis (only 39 cases), ethnicity, age, sex, lifestyle, other factors and the prognostic role of GPNMB in glioma requires further investigation. In the present study, it was revealed through data mining of the dataset from CGGA and subsequent validation with immunohistochemical staining of tissue microarray that high GPNMB expression, both at the mRNA and protein level, was associated with poor survival. Of note, multivariate analysis demonstrated that high GPNMB expression was an independent risk factor for patient survival. The difference in survival outcomes between patients with high and low GPNMB expression was not statistically significant in sub-populations aged $<30$ years, which may be due to the low percentage of patients with high GPNMB expression among young patients. In the present study, the population included in the survival analysis comprised Chinese patients, whereas the previous survival analysis was performed in American population (23), suggesting that the unfavorable prognostic effect of GPNMB on glioma may not be associated with ethnicity.

In conclusion, the results of the present study demonstrated that high GPNMB expression was associated with high malignancy and denoted unfavorable prognosis in patients with glioma. In addition, systematic bioinformatics analyses revealed that GPNMB expression was correlated with that of genes involved in hypoxia, angiogenesis, migration and invasion, which may be the potential mechanisms through which GPNMB mediates glioma progression.

\section{Acknowledgements}

Not applicable.

\section{Funding}

This study was funded by the National Natural Science Foundation of China (grant nos. 81803048 to XF and 81602663 to WT) and the Henan Provincial People's Hospital, People's Hospital of Zhengzhou University, People's Hospital of Henan University (grant nos. ZC20170016 and ZC20180135 to XF and ZC23456049 to LZ).

\section{Availability of data and materials}

The datasets used and/or analyzed in the present study are available from the corresponding author upon reasonable request.

\section{Authors' contributions}

XF, WT and SC conceived and designed the present study. XF, LZ, SK, TL, LH and PZ performed the research, collected and analyzed the data, interpreted the results and wrote the manuscript. All authors read and approved the final manuscript.

\section{Ethics approval and consent to participate}

The present study was approved by the Ethics Committee of Henan Provincial People's Hospital. 


\section{Patient consent for publication}

Not applicable.

\section{Competing interests}

The authors declare that they have no competing interests.

\section{References}

1. Ostrom QT, Gittleman H, Truitt G, Boscia A, Kruchko C and Barnholtz-Sloan JS: CBTRUS statistical report: Primary brain and other central nervous system tumors diagnosed in the United States in 2011-2015. Neuro Oncol 20 (Suppl 4): iv1-iv86, 2018.

2. Lapointe S, Perry A and Butowski NA: Primary brain tumours in adults. Lancet 392: 432-446, 2018.

3. Ostrom QT, Cote DJ, Ascha M, Kruchko C and BarnholtzSloan JS: Adult glioma incidence and survival by race or ethnicity in the United States from 2000 to 2014. JAMA Oncol 4: 1254-1262, 2018.

4. Hartmann C, Hentschel B, Wick Wm, Capper D, Felsberg J, Simon M, Westphal M, Schackert G, Meyermann R, Pietsch T, et al: Patients with IDH1 wild type anaplastic astrocytomas exhibit worse prognosis than IDH1-mutated glioblastomas, and IDH1 mutation status accounts for the unfavorable prognostic effect of higher age: Implications for classification of gliomas. Acta Neuropathol 120: 707-718, 2010.

5. Metellus P, Coulibaly B, Colin C, de Paula AM, Vasiljevic A, Taieb D, Barlier A, Boisselier B, Mokhtari K, Wang XW, et al: Absence of IDH mutation identifies a novel radiologic and molecular subtype of WHO grade II gliomas with dismal prognosis. Acta Neuropathol 120: 719-729, 2010.

6. Frattini V, Trifonov V, Chan JM, Castano A, Lia M, Abate F, Keir ST, Ji AX, Zoppoli P, Niola F, et al: The integrated landscape of driver genomic alterations in glioblastoma. Nat Genet 45 $1141-1149,2013$.

7. Ceccarelli M, Barthel FP, Malta TM, Sabedot TS, Salama SR, Murray BA, Morozova O, Newton Y, Radenbaugh A, Pagnotta SM, et al: Molecular profiling reveals biologically discrete subsets and pathways of progression in diffuse glioma. Cell 164: 550-563, 2016

8. Weterman MA, Ajubi N, van Dinter IM, Degen WG, van Muijen GN, Ruitter DJ and Bloemers HP: nmb, a novel gene, is expressed in low-metastatic human melanoma cell lines and xenografts. Int J Cancer 60: 73-81, 1995.

9. Zhuo H and Zhou L: Gpnmb/osteoactivin: An indicator and therapeutic target in tumor and nontumorous lesions. Pharmazie 71: $555-561,2016$.

10. Maric G, Annis MG, Dong Z, Rose AA, Ng S, Perkins D, MacDonald PA, Ouellet V, Russo C and Siegel PM: GPNMB cooperates with neuropilin-1 to promote mammary tumor growth and engages integrin $\alpha 5 \beta 1$ for efficient breast cancer metastasis. Oncogene 34: 5494-5504, 2015.

11. Li Y, Yuan S, Liu J, Wang Y, Zhang Y, Chen X and Si W: CSE1L silence inhibits the growth and metastasis in gastric cancer by repressing GPNMB via positively regulating transcription factor MITF. J Cell Physiol 235: 2071-2079, 2020.

12. Torres C, Linares A, Alejandre MJ, Palomino-Morales R, Martin M, Delgado JR, Martinez J and Perales S: The potential role of the glycoprotein osteoactivin/glycoprotein nonmetastatic melanoma protein B in pancreatic cancer. Pancreas 44: 302-310, 2015.

13. Taya M and Hammes SR: Glycoprotein non-metastatic melanoma protein B (GPNMB) and cancer: A novel potential therapeutic target. Steroids 133: 102-107, 2018.

14. Hoashi T, Sato S, Yamaguchi Y, Passeron T, Tamaki K and Hearing VJ: Glycoprotein nonmetastatic melanoma protein B, a melanocytic cell marker, is a melanosome-specific and proteolytically released protein. FASEB J 24: 1616-1629, 2010.

15. Abdelmagid SM, Barbe MF, Rico MC, Salihoglu S, Arango-Hisijara I, Selim AH, Anderson MG, Owen TA, Popoff SN and Safadi FF: Osteoactivin, an anabolic factor that regulates osteoblast differentiation and function. Exp Cell Res 314: 2334-2351, 2008.
16. Tomihari M, Hwang SH, Chung JS, Cruz PD Jr and Ariizumi K: $\mathrm{Gpnmb}$ is a melanosome-associated glycoprotein that contributes to melanocyte/keratinocyte adhesion in a RGD-dependent fashion. Exp Dermatol 18: 586-595, 2009.

17. Ripoll VM, Meadows NA, Raggatt LJ, Chang MK, Pettit AR, Cassady AI and Hume DA: Microphthalmia transcription factor regulates the expression of the novel osteoclast factor GPNMB. Gene 413: 32-41, 2008.

18. Sheng MH, Wergedal JE, Mohan S and Lau KH: Osteoactivin is a novel osteoclastic protein and plays a key role in osteoclast differentiation and activity. FEBS Lett 582: 1451-1458, 2008.

19. Ripoll VM, Irvine KM, Ravasi T, Sweet MJ and Hume DA: Gpnmb is induced in macrophages by IFN-gamma and lipopolysaccharide and acts as a feedback regulator of proinflammatory responses. J Immunol 178: 6557-6566, 2007.

20. Ahn JH, Lee Y, Jeon C, Lee SJ, Lee BH, Choi KD and Bae YS: Identification of the genes differentially expressed in human dendritic cell subsets by cDNA subtraction and microarray analysis. Blood 100: 1742-1754, 2002.

21. Chen C, Okita Y, Watanabe Y, Abe F, Fikry MA, Ichikawa Y, Suzuki H, Shibuya A and Kato M: Glycoprotein nmb is exposed on the surface of dormant breast cancer cells and induces stem cell-like properties. Cancer Res 78: 6424-6435, 2018.

22. Oyewumi MO, Manickavasagam D, Novak K, Wehrung D, Paulic N, Moussa FM, Sondag GR and Safadi FF: Osteoactivin (GPNMB) ectodomain protein promotes growth and invasive behavior of human lung cancer cells. Oncotarget 7: 13932-13944, 2016.

23. Kuan CT, Wakiya K, Dowell JM, Herndon JE II, Reardon DA, Graner MW, Riggins GJ, Wikstrand CJ and Bigner DD: Glycoprotein nonmetastatic melanoma protein B, a potential molecular therapeutic target in patients with glioblastoma multiforme. Clin Cancer Res 12: 1970-1982, 2006.

24. Ono Y, Chiba S, Yano H, Nakayama N, Saio M, Tsuruma K, Shimazawa M, Iwama $\mathrm{T}$ and Hara H: Glycoprotein nonmetastatic melanoma protein $\mathrm{B}$ (GPNMB) promotes the progression of brain glioblastoma via $\mathrm{Na}^{+} / \mathrm{K}^{+}$-ATPase. Biochem Biophys Res Commun 481: 7-12, 2016.

25. Bao G, Wang N, Li R, Xu G, Liu P and He B: Glycoprotein non-metastaticmelanoma protein $\mathrm{B}$ promotes glioma motility and angiogenesis through the Wnt $/ \beta$-catenin signaling pathway. Exp Biol Med (Maywood) 241: 1968-1976, 2016.

26. Reifenberger G, Weber RG, Riehmer V, Kaulich K, Willscher E, Wirth H, Gietzelt J, Hentschel B, Westphal M, Simon M, et al: Molecular characterization of long-term survivors of glioblastoma using genome- and transcriptome-wide profiling. Int J Cancer 135: 1822-1831, 2014.

27. Yan W, Zhang W, You G, Zhang J, Han L, Bao Z, Wang Y, Liu Y, Jiang C, Kang C, et al: Molecular classification of gliomas based on whole genome gene expression: A systematic report of 225 samples from the Chinese glioma cooperative group. Neuro Oncol 14: 1432-1440, 2012.

28. R Core Team. R: A language and environment for statistical computing. R Foundation for Statistical Computing, Vienna, Austria. URL http://www.R-project.org/, 2014.

29. Wettenhall JM and Smyth GK: limmaGUI: A graphical user interface for linear modeling of microarray data. Bioinformatics 20: 3705-3706, 2004.

30. Yu G, Wang LG, Han Y and He QY: clusterProfiler: An R package for comparing biological themes among gene clusters. OMICS 16: 284-287, 2012.

31. Luo W and Brouwer C: Pathview: An R/Bioconductor package for pathway-based data integration and visualization. Bioinformatics 29: 1830-1831, 2013.

32. Verhaak RG, Hoadley KA, Purdom E, Wang V, Qi Y, Wilkerson MD, Miller CR, Ding L, Golub T, Mesirov JP, et al: Integrated genomic analysis identifies clinically relevant subtypes of glioblastoma characterized by abnormalities in PDGFRA, IDH1, EGFR, and NF1. Cancer Cell 17: 98-110, 2010

33. Hofer S and Lassman AB: Molecular markers in gliomas: Impact for the clinician. Target Oncol 5: 201-210, 2010.

34. Ma R, de Pennington N, Hofer M, Blesing C and Stacey R: Diagnostic and prognostic markers in gliomas-an update. Br J Neurosurg 27: 311-315, 2013.

35. Thomas L, Di Stefano AL and Ducray F: Predictive biomarkers in adult gliomas: The present and the future. Curr Opin Oncol 25: 689-694, 2013

36. Ellison DW: Multiple molecular data sets and the classification of adult diffuse gliomas. N Engl J Med 372: 2555-2557, 2015.

37. Gimona $\mathrm{M}$ and Buccione R: Adhesions that mediate invasion. Int J Biochem Cell Biol 38: 1875-1892, 2006. 
38. Yamaguchi $\mathrm{H}$, Wyckoff $\mathrm{J}$ and Condeelis $\mathrm{J}$ : Cell migration in tumors. Curr Opin Cell Biol 17: 559-564, 2005.

39. Kedrin D, van Rheenen J, Hernandez L, Condeelis J and Segall JE: Cell motility and cytoskeletal regulation in invasion and metastasis. J Mammary Gland Biol Neoplasia 12: 143-152, 2007.

40. Staff AC: An introduction to cell migration and invasion. Scand J Clin Lab Invest 61: 257-268, 2001.

41. Sciumè G, Santoni A and Bernardini G: Chemokines and glioma: Invasion and more. J Neuroimmunol 224: 8-12, 2010.

42. Wick W, Naumann U and Weller M: Transforming growth factor-beta: A molecular target for the future therapy of glioblastoma. Curr Pharm Des 12: 341-349, 2006.

43. Wells A, Grahovac J, Wheeler S, Ma B and Lauffenburger D Targeting tumor cell motility as a strategy against invasion and metastasis. Trends Pharmacol Sci 34: 283-289, 2013.

44. Laufs S, Schumacher J and Allgayer H: Urokinase-receptor (u-PAR): An essential player in multiple games of cancer: A review on its role in tumor progression, invasion, metastasis, proliferation/dormancy, clinical outcome and minimal residual disease. Cell Cycle 5: 1760-1771, 2006.

45. Kessenbrock K, Plaks V and Werb Z: Matrix metalloproteinases: Regulators of the tumor microenvironment. Cell 141: 52-67, 2010.

46. Kassis J, Lauffenburger DA, Turner T and Wells A: Tumor invasion as dysregulated cell motility. Semin Cancer Biol 11: 105-117, 2001.

47. Jiang WG, Sanders AJ, Katoh M, Ungefroren H, Gieseler F, Prince M, Thompson SK, Zollo M, Spano D, Dhawan P, et al: Tissue invasion and metastasis: Molecular, biological and clinical perspectives. Semin Cancer Biol 35 (Suppl): S244-S275, 2015.

48. Jakowlew SB: Transforming growth factor-beta in cancer and metastasis. Cancer Metastasis Rev 25: 435-457, 2006.

49. Friedl $\mathrm{P}$ and Wolf $\mathrm{K}$ : Tumour-cell invasion and migration: Diversity and escape mechanisms. Nat Rev Cancer 3: 362-374, 2003.

50. Andreasen PA, Kjøller L, Christensen L and Duffy MJ: The urokinase-type plasminogen activator system in cancer metastasis: A review. Int J Cancer 72: 1-22, 1997.

51. Westermarck $\mathbf{J}$ and Kähäri VM: Regulation of matrix metalloproteinase expression in tumor invasion. FASEB J 13: 781-792, 1999.
52. Jain RK, di Tomaso E, Duda DG, Loeffler JS, Sorensen AG and Batchelor TT: Angiogenesis in brain tumours. Nat Rev Neurosci 8: 610-622, 2007.

53. Anderson SA, Glod J, Arbab AS, Noel M, Ashari P, Fine HA and Frank JA: Noninvasive MR imaging of magnetically labeled stem cells to directly identify neovasculature in a glioma model. Blood 105: 420-425, 2005.

54. Smith SJ, Tilly H, Ward JH, Macarthur DC, Lowe J, Coyle B and Grundy RG: CD105 (Endoglin) exerts prognostic effects via its role in the microvascular niche of paediatric high grade glioma. Acta Neuropathol 124: 99-110, 2012.

55. Hudson AL, Parker NR, Khong P, Parkinson JF, Dwight T, Ikin RJ, Zhu Y, Chen J, Wheeler HR and Howell VM: Glioblastoma Recurrence correlates with increased APE1 and polarization toward an immuno-suppressive microenvironment. Front Oncol 8: 314, 2018.

56. Walentynowicz KA, Ochocka N, Pasierbinska M, Wojnicki K, Stepniak K, Mieczkowski J, Ciechomska IA and Kaminska B: In search for reliable markers of glioma-induced polarization of microglia. Front Immunol 9: 1329, 2018.

57. Sun X, Liu X, Xia M, Shao Y and Zhang XD: Multicellular gene network analysis identifies a macrophage-related gene signature predictive of therapeutic response and prognosis of gliomas. J Transl Med 17: 159, 2019.

58. Zhou W, Ke SQ, Huang Z, Flavahan W, Fang X, Paul J, Wu L, Sloan AE, McLendon RE, Li X, et al: Periostin secreted by glioblastoma stem cells recruits M2 tumour-associated macrophages and promotes malignant growth. Nat Cell Biol 17: 170-182, 2015.

59. Hambardzumyan D, Gutmann DH and Kettenmann H: The role of microglia and macrophages in glioma maintenance and progression. Nat Neurosci 19: 20-27, 2016.

60. Mantovani A, Sozzani S, Locati M, Allavena P and Sica A: Macrophage polarization: Tumor-associated macrophages as a paradigm for polarized M2 mononuclear phagocytes. Trends Immunol 23: 549-555, 2002.

(c) (i) () This work is licensed under a Creative Commons Attribution-NonCommercial-NoDerivatives 4.0 International (CC BY-NC-ND 4.0) License. 